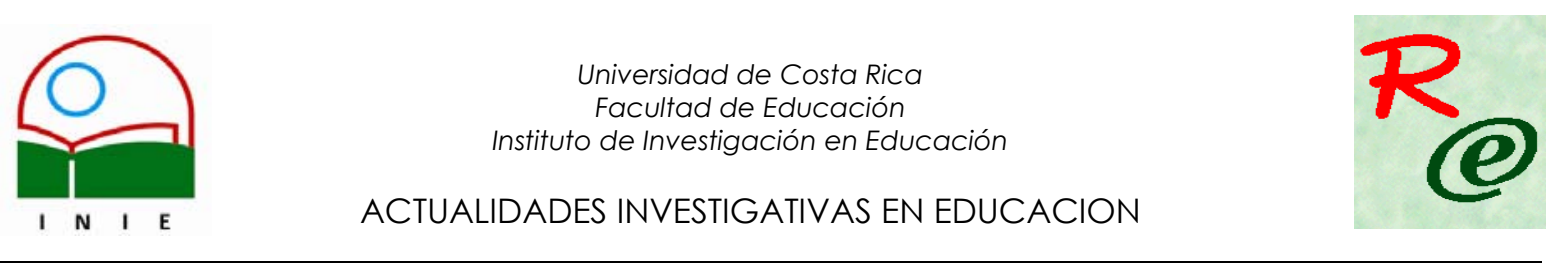

\title{
CONSIDERACIONES PARA UNA EVALUACIÓN DOCENTE EN LA UNIVERSIDAD DE COSTA RICA
}

\author{
Alicia Vargas Porras ${ }^{1}$ \\ Ma. Lourdes Calderón Laguna ${ }^{2}$
}

Resumen: En este artículo se presentan los resultados de un proyecto de investigación realizado en el $2002-$ 2003 por Alicia E. Vargas, Carolina Bolaños, José Miguel Gutiérrez, Lorena Kikut, María Lourdes Calderón y Miguel Ángel González, docentes investigadores del Centro de Evaluación Académica de la Universidad de Costa Rica. El objetivo principal fue identificar características del profesorado universitario y de su trabajo, como insumos para mejorar la evaluación docente que se realiza en esta Universidad. La investigación se centró en construir un concepto de docencia, en términos de las competencias y desempeños que se consideran como excelentes. Para ello, además de la obligada revisión bibliográfica sobre el tema, se llevó a cabo un estudio, mediante talleres y encuestas, para construir el concepto de docente excelente, según lo plantea el mismo profesorado, el alumnado y las directivas universitarias. En una segunda etapa del estudio, se abordó el tema de las especificidades didácticas, según tipos de curso, mediante consulta a la comunidad docente y talleres con amplia participación.

Los resultados obtenidos en esta investigación aportan una conceptualización de lo que significa ser docente en la UCR y, así mismo, se logra diferenciar los requerimientos del desempeño docente, según tipo de curso. La información generada aporta criterios para la construcción de evaluaciones docentes en el ámbito universitario.

Palabras clave: EVALUACIÓN DOCENTE/ LABOR DOCENTE/ COMPETENCIAS DOCENTES/ EVALUACIÓN POR TIPO DE CURSO/ TIPOS DE CURSO/ ESPECIFICIDADES DIDÁCTICAS/ PERFIL DEL DOCENTE/ EXCELENCIA DOCENTE/

Abstract: This article presents the results of a research carried out during the period 2002-2003 by Alicia E. Vargas, Carolina Bolaños, José Miguel Gutiérrez, Lorena Kikut, María Lourdes Calderón and Miguel Ángel González, researches of the Centre for Academic Evaluation of the University of Costa Rica. Its main objective was to identify teaching qualities in higher education as inputs for the improvement of the evaluation of teachers that is carried out at the University of Costa Rica. The study aimed, in the first place, to clarify the concept of an excellent teacher in terms of competencies and expected production at this university. In that respect, besides a thorough review of literature, workshops were carried out and questionnaires were applied in order to clarify the main aspects considered by teachers, students and university authorities in that respect. In a second stage of the study and with a wide participation of the university community, there was a clarification of the main didactic specialities that teachers must have, according to the different course modalities that they may use.

The generated information obtained in this research contributes to clarify the characteristics of an ideal teacher at the UCR and, at the same time, identifies the expected requirements according to the different type of courses. The information obtained brings an array of criteria that may be used in building up instruments of teacher's evaluations at the university level.

Key words: TEACHING EVALUATION/ TEACHING WORK/ TEACHING COMPETENCIES/ COURSE EVALUATION/ TYPE OF COURSES/ DIDACTIC SPECIFICATIONS/ TEACHER'S PROFILE/TEACHING EXCELLENCE/

\footnotetext{
1 Doctora en Educación; Licenciada en Ciencias de la Educación con énfasis en Administración Educativa ambos títulos de la Universidad de Costa Rica. Actualmente es Jefa del Departamento de Investigación y Evaluación Académica del Centro de Evaluación Académica, en donde ha laborado en los últimos 15 años. Es también docente de la Escuela de Administración Educativa.

Correo electrónico: aevargas@cariari.ucr.ac.cr

${ }^{2}$ Licenciada en Psicología por la Universidad de Costa Rica. Los últimos 15 años ha trabajado para el Centro de Evaluación Académica de la Universidad de Costa Rica. Correo electrónico: mlcalder@cariari.ucr.ac.cr
}

Artículo presentado: 14 de julio, 2005

Aprobado: 26 de setiembre, 2005 


\section{Presentación}

La Universidad de Costa Rica tiene a su haber, una larga trayectoria de evaluaciones del desempeño del personal docente en el aula, basada en una concepción general y homogénea de la enseñanza universitaria. Actualmente no cuenta con un sistema de evaluación que responda a diferentes fines, necesidades e intereses de la institución y que atienda las particularidades de la actividad docente, según lo exige la heterogeneidad de áreas profesionales y de conocimiento.

Diseñar un sistema de evaluación del desempeño docente que responda a tales propósitos, exigió, en un primer momento, resignificar el concepto de evaluación, a la luz de las prácticas existentes y de las propuestas actuales en el ámbito universitario; buscar consenso en la comunidad universitaria sobre lo que se debe entender por un desempeño docente de calidad y por último, articular procedimientos de evaluación y uso de los resultados, de modo que éstos sean válidos y oportunos para los implicados en los procesos de docencia: profesores, estudiantes, directivas y administración universitaria.

El artículo inicia con un marco teórico que incluye el concepto de evaluación desde donde se parte, continuando luego con una reflexión acerca del personal docente y los acontecimientos sociopolíticos en los albores del siglo XXI. Finalmente, se hace una síntesis de los resultados de la investigación la cual aporta una caracterización del desempeño del docente de calidad de la Universidad de Costa Rica y la aclaración de las competencias según diferentes tipos de cursos que se imparten en la institución, como referentes por considerar en la evaluación docente.

Es necesario mencionar que, para efectos de este artículo y como primera parte de la investigación realizada, únicamente se presentan una serie de insumos que se retomarán luego, en una segunda etapa de la investigación, al proponer diferentes formas de evaluación docente, según diversos fines, lo cual constituirá un sistema institucional de evaluación docente.

\section{Aproximación conceptual a la evaluación docente}

La investigación realizada se fundamentó en una amplia revisión bibliográfica, la cual indagó aspectos sobre la evaluación, la relación con la docencia universitaria, así como un 
análisis de la situación de la Universidad de Costa Rica ante los nuevos retos sociales, económicos y políticos relacionados con la evaluación del personal docente.

\subsection{El concepto de evaluación.}

Tradicionalmente, la evaluación se ha entendido como un proceso objetivo que busca aclarar aspectos de una realidad que se concibe como predecible, contabilizable, generalizable y, sobre todo libre de valores. En este sentido, la idea de evaluación se asocia al uso de parámetros, indicadores, métodos que promuevan la objetividad y la distancia entre lo que se evalúa y las personas involucradas en practicar la evaluación. Un ejemplo de esta concepción de evaluación son los exámenes de Bachillerato que se practican en Costa Rica al finalizar el cuarto ciclo de la enseñanza media, en donde se juzga el desempeño estudiantil a partir de una prueba estandarizada que ha llegado a ser considerada desafortunadamente- como el norte de la educación costarricense, ya que los temas que se incluyen en ese examen se privilegian por encima de cualquier otro aprendizaje, con lo cual la educación, asociada a este tipo de evaluación, se convierte en un ejercicio de transmisión de conocimientos y de memorización de contenidos. Así mismo, es común la evaluación docente a partir de un cuestionario generalizado, en donde se estandariza el quehacer del personal docente y se intenta medir su desempeño mediante indicadores y parámetros normalizadores.

Hoy en día, sin embargo, la teoría de la evaluación está siendo reconstruida más allá de este enfoque tradicional, en el entendido de que "la realidad" es dinámica, diversa, compleja, cambiante, subjetiva. De ahí que la evaluación se conciba como un proceso más reflexivo y menos informativo, en donde se incorporan los valores, las preocupaciones y las concepciones iniciales de las personas involucradas, así como el contexto histórico y las situaciones en donde suceden los hechos que se evalúan. Como indica De Alba (1991, 1993) la evaluación es un proceso reflexivo que considera tanto el origen y desarrollo de un proceso dado o situación, como sus características estructurales. Demanda la consideración histórica (origen, desarrollo, futuro posible) y la comprensión de las "condiciones estables" (normas y regulaciones), así como las diferentes formas mediante las cuales los diferentes actores asumen las condiciones dadas. En este sentido, la comprensión de lo que está siendo evaluado sólo puede realizarse con la participación de las personas involucradas, en un análisis de las diferencias y contradicciones, compromisos y responsabilidades, conflictos y posibilidades. 
Más que entender la evaluación como un instrumento de control externo sobre lo que se hace y cómo se hace, los nuevos enfoques de evaluación la conciben como un proceso que permite aprender sobre la realidad en la que se actúa y que, por lo tanto tiene propósitos formativos. En el caso de la Universidad de Costa Rica, el interés por establecer un sistema de evaluación docente, surge de la necesidad de trascender esa idea de control y de información, para incorporar además, procesos de evaluación que sean formativos que puedan ser realizados ( $\mathrm{y}$ controlados) a lo interno de las unidades académicas y orientados a procesos de mejora.

La evaluación realizada por agentes externos y orientada por un núcleo de criterios e indicadores comunes al profesorado universitario puede ser útil para algunos efectos institucionales como los de promoción (ascenso en Régimen Académico) y como información general. Pero a la vez se hace necesario incorporar, en un sistema de evaluación docente, otras formas de evaluar con base en enfoques participativos y con propósitos formativos, con el fin de promover la autorreflexión y el autoaprendizaje de personas y unidades académicas.

\subsection{La labor docente.}

En un intento por comprender la docencia y la labor que realiza el personal docente universitario, se pueden contraponer dos enfoques diferentes. El primero se basa en una aproximación técnica y academicista de la profesión docente, sustentada en la lógica de la razón instrumental, la cual asigna al profesor la función básica de transmitir contenidos. Desde esta perspectiva, como aclaran Díaz-Barriga y Pacheco-Méndez (1997), se espera que las docentes y los docentes procuren la transmisión, utilización y legitimación de conocimiento científico, el cual ha sido elaborado y escogido por especialistas externos como "currículum oficial". De esta forma, el carácter ético, político, inacabado y contingente de la formación humana, es ignorado.

Dentro del enfoque técnico-academicista previamente planteado, es posible una evaluación del personal docente fundamentada en criterios eficientistas, centrada en los productos, número de publicaciones, grado académico, calificación de sus alumnos. Este enfoque atiende más a los resultados que al proceso y deja por fuera aspectos fundamentales del quehacer docente, tales como aquellos relacionados con la producción de bienes simbólicos y situaciones institucionales y colectivas que determinan el quehacer 
docente. De esta forma, la evaluación se puede volver un proceso solitario, individual, con fines controladores y políticos, que uniforman la labor docente, con criterios de productividad y rendimiento.

Sin embargo, en los últimos veinte años han venido cobrando fuerza una serie de propuestas constructivistas y críticas de la función docente, en donde se busca fortalecer la reflexión de los docentes sobre su práctica misma, en un contexto determinado. Se fortalece el vínculo de la investigación y se concibe al docente como un profesional reflexivo, que trasciende los muros de la institución para analizar y develar todo tipo de intereses que subyacen a los procesos educativos, así como un acercamiento a la comprensión de la realidad social, con el ánimo de buscar la emancipación de las personas. Al respecto, autores como Giroux (1997) y Apple (1996) conciben a los educadores como "trabajadores culturales", intelectuales con una relativa autonomía estructural, que son capaces de ejercer cierta dirección cultural en los diferentes grupos con quienes trabajan, para provocar la transformación social e impulsar prácticas democráticas que conlleven a la comprensión crítica de las sociedades y su desarrollo.

En la Universidad de Costa Rica ha prevalecido una evaluación docente que promueve un marco de simpleza técnica, con pretensiones de neutralidad valorativa y creyéndola desvinculada de las fuerzas políticas. Se ha dado así, en la práctica, una evaluación tecnocrática, caracterizada por el interés de conocer los resultados que se traslucen en el producto (desempeño docente) y que no ha permitido incidir en mejores formas de realizar la función docente.

Considerando las nuevas tendencias de evaluación docente, corresponde ubicarnos en un enfoque que abandone el concepto del profesor tradicional académico y enciclopedista, y el del experto técnico, cuya función principal es transmitir conocimientos mediante la aplicación rutinaria de procedimientos de instrucción diseñados y ofrecidos desde afuera. Se hace necesario privilegiar un papel más activo del profesorado en el diseño, desarrollo, seguimiento y reformulación de estrategias pedagógicas, en el cual la evaluación cobra importancia, en tanto proceso reflexivo que permite el autoanálisis de la labor docente y la reflexión de su quehacer con miras a su mejor desempeño. En ese sentido, se privilegia la idea de autoevaluación autogestionaria. 
Esta concepción de docente como profesional requiere considerar la compleja labor de incorporar la docencia, la investigación y la acción social como dinamizadores pedagógicos del proceso educativo que se realiza en la Universidad de Costa Rica y, con el fin de clarificar aspectos por considerar en una evaluación docente, fue necesario identificar una serie de competencias relacionadas con el quehacer docente institucional. Para ello se utilizó un esquema genérico (Scriven 1997; Jiménez 1999) que permite categorizar el conjunto de atributos que la comunidad universitaria señaló como propios de una buena docencia, según las siguientes dimensiones:

$\checkmark$ qué debe saber el docente (conocimientos)

$\checkmark$ qué debe saber hacer (método, tecnologías, técnicas, competencias didácticas)

$\checkmark$ cómo debe ser y comportarse (relaciones, habilidades sociales, actitudes personales)

\section{Caracterización del personal docente universitario.}

Como primera etapa para el diseño de un sistema de evaluación docente que considere tanto los fines de información como los de formación, se hizo necesario aclarar las competencias generales requeridas del quehacer docente en el contexto universitario en el entendido de que, al desarrollar las particularidades de un sistema de evaluación docente con diferentes fines, se hará necesario afinar dichas competencias.

Considerando los documentos oficiales de la Universidad, las opiniones de estudiantes y docentes, lo dispuesto en documentos de varias instancias universitarias ${ }^{3}$ y la revisión de literatura en el campo correspondiente, se llegó a caracterizar al profesor y profesora universitaria, a partir de las tres dimensiones arriba señaladas.

Para comprender estas dimensiones tanto en términos conceptuales como en relación con la importancia que tienen en el establecimiento de un sistema de calidad docente, a continuación se aclara cada una de ellas, incluyendo no sólo su identificación sino también cómo se pueden comprobar y su importancia. ${ }^{4}$

\footnotetext{
${ }^{3}$ Centro de Evaluación Académica, Departamento de Docencia Universitaria.

${ }^{4}$ En la investigación realizada, cada uno de los aspectos considerados en las tres dimensiones se trabajaron hasta el nivel de "criterios", lo cual permite construir evaluaciones docentes. Por razones de espacio no se incluyen en el presente artículo pero mayor información puede ser obtenida en el Centro de Evaluación Académica de la UCR: cea@cariari.ucr.ac.cr
} 


\section{a) Primera dimensión: ¿Qué debe saber?}

Esta dimensión agrupa un conjunto de competencias relativas al conocimiento y preparación académica deseable en todo docente. Se diferencia entre los conocimientos disciplinares, relativos a las temáticas de los cursos que tiene a cargo y los conocimientos pedagógicos y didácticos, relativos a la forma en que tales conocimientos se enseñan, se aprenden y se evalúa su aprendizaje. En esta dimensión se puede pensar en competencias como las siguientes:

\section{- Dominio de su campo de acción:}

Esta competencia incluye aspectos relacionados con la preparación académica del profesor y la profesora en el área disciplinar en la que ejerce la docencia, aspecto de exigencia básica para el ejercicio docente. Se evalúa considerando los atestados personales: certificados de estudios formales, estudios no formales, investigaciones publicadas, producción académica, participaciones en eventos de su comunidad científica, experiencia relevante en el ejercicio de su profesión. Se comprueba mediante documentos. No es relevante incluirlo en preguntas de cuestionarios o de otro tipo.

Tiene importancia para la contratación, para las políticas de desarrollo profesional (estudios de postgrado del profesorado, becas), para las políticas de producción investigativa y para ascenso en Régimen Académico ${ }^{5}$. Constituye la base para asegurar la pertinencia, actualización y alto nivel del acerbo de conocimientos disciplinares que se producen y circulan en una unidad académica.

\section{- Conocimiento del campo de la pedagogía y la didáctica universitaria:}

Esta competencia es exigencia básica para el ejercicio docente. El personal docente debe conocer teoría de la educación y del currículo, así como las propuestas didácticas actuales y los métodos de evaluación. Se evalúa mediante certificación de estudios y cursos sobre pedagogía y didáctica. Se manifiesta en la práctica docente y es objeto de evaluación indirecta al evaluar el ejercicio práctico de la docencia, bajo el supuesto de que tales conocimientos tienen sentido si quienes ejercen la docencia los aplican en el aula. Se

\footnotetext{
${ }^{5}$ El Régimen Académico de la Universidad de Costa Rica es el sistema que organiza al profesorado universitario en categorías, con base en sus méritos académicos y en su experiencia universitaria. (Artículo 1, Reglamento de Régimen Académico y Servicio Docente). 
manifiesta también en la participación y aportes del profesorado al mantenimiento y actualización del currículo mediante el diseño de los programas de asignaturas.

Tiene importancia para los procesos de mejoramiento de la calidad de la docencia y las políticas de desarrollo pedagógico del profesorado.

\section{- Normativa institucional:}

Es una exigencia corporativa. El personal docente debe conocer la universidad, su vocación social expresada en la declaración de principios; las normas universitarias, sus deberes y derechos; sus obligaciones contractuales; las costumbres y formas de hacer de la comunidad universitaria. Se evalúa mediante juicio de los demás miembros del colectivo, principalmente del superior inmediato. El estudiantado puede informar sobre los aspectos de cumplimiento por el personal docente de las normas y compromisos relacionados con el ejercicio de la docencia.

Tiene importancia para mantener un clima organizacional sano, para renovación de contratos y como criterio para la toma de decisiones relacionada con incentivos.

\section{b) Segunda dimensión: ¿Qué debe saber hacer?}

Esta segunda dimensión abarca el conjunto de competencias prácticas que el personal docente pone en juego durante su intervención didáctica; se refiere a lo que sucede en el aula de clase y en las interrelaciones entre docentes y estudiantes.

\section{- Planificación de la enseñanza:}

El personal docente debe ser capaz de programar los cursos a su cargo, lo cual comprende: la formulación concreta de lo que va a enseñar, en términos de contenidos y de objetivos de aprendizaje; la secuencia y distribución en el tiempo disponible de los temas y actividades; la definición de las actuaciones que le corresponderán, de las actividades que realizará el estudiantado y de los recursos con que se cuenta para llevarlas a cabo; los procedimientos de evaluación de los aprendizajes y la definición de los aspectos que utilizará para juzgar los resultados obtenidos por el estudiantado. La programación debe incluir estrategias para comunicar al estudiantado la propuesta de trabajo académico y para permitir su participación en la definición específica de aspectos operativos y metodológicos. La calidad de la planificación se evalúa atendiendo tres aspectos: la coherencia de los 
contenidos y objetivos con el currículo; el equilibrio didáctico en términos de adecuación y variedad de métodos y evaluaciones; la funcionalidad del programa para permitir al estudiantado comprender lo que tiene que aprender, lo que debe hacer para ello y estar bien informado y orientado durante todo el curso. Los aspectos curriculares se comprueban mediante análisis del documento de programación por la persona que coordina el área curricular correspondiente. Los aspectos de adecuación didáctica y funcionalidad se comprueban mediante la percepción y el juicio del estudiantado y mediante procesos de autoevaluación por los propios profesores y profesoras.

La planificación de la enseñanza tiene importancia para la administración del currículo y permite juzgar el conocimiento pedagógico del personal docente y su capacidad de proyectarlo en su práctica.

\section{- Desarrollo de lecciones aplicando estrategias didácticas:}

El ejercicio de la docencia abarca un conjunto amplio de competencias ejercidas por el personal docente con el objeto de mediar entre quien aprende y el conocimiento que se espera producir. La enseñanza efectiva exige al profesorado tener la habilidad para encontrar la combinación de métodos más adecuada a la naturaleza de lo que enseña y a las características de la población estudiantil. El problema de las especificidades didácticas asociadas con la naturaleza de las carreras y tipos de cursos serán objeto de evaluación específica.

En este apartado se enumeran los aspectos que fueron considerados comunes al ejercicio docente en general, a saber:

- El empleo de distintas estrategias y métodos, como exposiciones claras, uso de medios, organización de trabajos grupales, discusiones, estudio de casos, prácticas, trabajo individual, trabajo colaborativo.

- La capacidad de facilitar la motivación del estudiantado: referida sobre todo al grado de interés que el docente o la docente despierta y al sentido de auto eficacia que promueve en sus estudiantes.

- La capacidad de adecuar su enseñanza: esto significa que debe modificar su intervención pedagógica si así lo exigen las necesidades, expectativas e intereses del estudiantado. Para ello tiene que darse cuenta del tipo de procesos y formas de 
comprensión que el estudiantado pone en juego y aceptar sus aportes y puntos de vista.

- Dar gran importancia a la práctica de manera que quien aprende pueda aplicar el conocimiento fuera del aula en situaciones propias del entorno nacional.

- Integrar estrategias de investigación formativa, que fomenten un espíritu de indagación y de creatividad.

- La medida en que centra su acción en el estudiantado para que aprenda a aprender: el personal docente ha de ser capaz de considerar al estudiantado como centro del proceso de enseñanza y aprendizaje y esforzarse por conocer la forma en que aprenden sus estudiantes y abordan las actividades que propone; más que exigir dar cuenta exacta de los contenidos, la enseñanza universitaria eficaz debe centrarse en facilitar el surgimiento de aspectos propios y de habilidades de pensamiento de orden superior.

Todos estos aspectos se comprueban averiguando la percepción y el juicio del estudiantado sobre la intervención del personal docente $\mathrm{y}$, desde luego, mediante la autoevaluación que el profesorado mismo haga de su propia labor.

El ejercicio docente constituye en sí mismo la calidad de la docencia. Su evaluación tiene la mayor importancia para todos los procesos de mejora o aseguramiento de la calidad, para la formación y profesionalización del profesorado y para la toma de decisiones departamentales.

\section{- Seguimiento y evaluación de los aprendizajes:}

La evaluación de los aprendizajes que realiza el personal docente, para cumplir con su función tradicional de dar cuenta mediante calificaciones de los resultados de aprendizaje, debe ser una evaluación ajustada a los requerimientos curriculares y administrativos, ser realizada con justicia y estar diseñada cumpliendo los requisitos técnicos propios del tipo de instrumentos que se utilicen. Además, los procedimientos de seguimiento y evaluación deben tener una intención formativa, como una estrategia enfocada a estimular una actitud analítica y crítica en el estudiantado y facilitar el proceso de controlar el propio proceso de aprender. 
La consulta al estudiantado sobre los procedimientos de evaluación que utiliza el docente o la docente es sólo una manera de comprobar qué también él o ella evalúa al estudiantado. Esto nos ayuda a identificar aspectos particulares de un curso que merecen atención. Sin embargo, hay otras formas válidas para evaluar este aspecto, como son mediante el análisis de los instrumentos utilizados, comprobación de las realizaciones del estudiantado, uso de los resultados, reportes de observaciones de clase de otros colegas y análisis del sistema de calificaciones utilizado.

Esta última forma de comprobación, poco usual, tiene que formar parte de procesos auto evaluativos en los que se implique al propio profesor o profesora con intenciones de mejorar su práctica. Esta competencia tiene importancia para controlar el cumplimiento de normas por parte del o la docente, para el mejoramiento de la calidad docente y para la autoevaluación del currículo que realmente se ejecute, pues la evaluación refleja lo que se exige al estudiantado y los criterios con que se juzgan sus resultados de aprendizaje, constituyendo el criterio más seguro para juzgar la calidad del currículo ejecutado, frente al diseñado.

\section{- Investigación:}

En principio, la investigación debe formar parte del desempeño del personal docente. Parece conveniente diferenciar la investigación formal en el ámbito de las disciplinas, de la investigación sobre temas relacionados con cómo enseñarla o cómo se aprende, es decir, la investigación en didácticas específicas. La medida en que se exija al personal docente realizar investigaciones como parte de sus funciones dependerá de los temas en que se desempeña, de las políticas de la unidad, de su dedicación a la docencia. Es necesario promover la importancia de la investigación asociada con la función de enseñar, prácticamente ausente en nuestra universidad; en este sentido se deberá considerar como labor investigativa la ejecución de proyectos de aula en los que se aplique una metodología específica, con fundamento teórico, destinada a mejorar probadamente los procesos didácticos. Las competencias investigativas del personal docente se comprueban exclusivamente mediante análisis de los resultados que, dependiendo de las prácticas de su comunidad científica, deben ser difundidos y publicados en medios reconocidos.

La producción investigativa tiene importancia para mantener el prestigio académico, para incorporar al currículo los avances del conocimiento producido y muy particularmente 
para hacer posible que el personal docente pueda hacer partícipes a sus estudiantes de los procesos de investigación que lleva a cabo con fines de formar en ellos las competencias y actitudes propias de un investigador.

\section{- Cumplimiento de la normativa universitaria:}

El personal docente ha de conocer y cumplir los compromisos contractuales y las normas de convivencia académica propias de la universidad. La comprobación de este cumplimiento tiene sentido cuando los resultados se pueden utilizar para tomar decisiones, bien sea de incentivos o de sanciones. Por tanto debería ser función del superior jerárquico inmediato, aun cuando parte de la información relativa al cumplimiento de normas puede obtenerse solicitándola al estudiantado.

Esta competencia tiene importancia para la toma de decisiones sobre contratación, renovación de contratos, incentivos y sanciones.

\section{c) Tercera dimensión: ¿Cómo debe ser?}

Esta última dimensión supone una serie de competencias requeridas para una docencia excelente, la cual exige un conjunto de actitudes propias, identificables y por tanto reconocibles en el comportamiento del personal docente, cuya presencia debe contar con un reconocimiento en términos de incentivos y de sanciones. Las últimas investigaciones sobre calidad docente muestran, sin lugar a dudas, que en todas las disciplinas y todas las latitudes, el estudiantado considera las competencias relacionadas con las actitudes como más deseables en sus profesores y profesoras que aquellas relativas al conocimiento profundo e incluso a las meras destrezas metodológicas utilizadas en la docencia.

Los aspectos principales que abarca esta dimensión tienen que ver, sobre todo, con el respeto en el trato al estudiantado, la apertura intelectual, no abusar del poder que otorga el estatus de docente, la disponibilidad de atender al estudiantado, dentro y fuera del aula, la preocupación por facilitar su aprendizaje y la consiguiente actitud de adaptar la propia enseñanza a las necesidades del estudiantado y, en general, la calidad del clima en que se desarrolla un curso. Igualmente, al profesorado se le exige un alto nivel de conciencia social y de su función universitaria, que se muestre en actitudes de responsabilidad científica y de proyección social. 
La comprobación de estos atributos se consigue a partir de la información que brinda el estudiantado, y puede además triangularse con información aportada por colegas y por la o el superior jerárquico.

Su valoración tiene sentido tanto para la toma de decisiones como, sobre todo, para el mejoramiento, cuando los y las docentes asuman la evaluación como uno de los mecanismos idóneos para la autorregulación.

\section{En resumen, los aspectos específicos relacionados con cada una de las tres dimensiones anteriormente mencionadas son los siguientes:}

\section{¿Qué debe saber?}

- Dominio de su campo de conocimiento

- Principios teóricos y epistemológicos de la educación

- Principios de planificación curricular: general y didáctica

- Los procesos de enseñanza y aprendizaje en el nivel universitario

- Estrategias didácticas para el aprendizaje en la universidad

- Evaluación de los aprendizajes a nivel universitario

- Normativa universitaria

\section{¿Qué debe saber hacer?:}

- Planificación de la enseñanza: organizar un curso, elaborar un programa de curso

- Uso del programa para orientar el curso y las lecciones

- Selección de materiales para el aprendizaje (lecturas, casos, prácticas, instrumentos)

- Estrategias didácticas para el aprendizaje

- Desarrollo de las lecciones

- Mediación pedagógica (entre sus estudiantes y el objeto por aprender)

- Diseño y elaboración de estrategias de evaluación de los aprendizajes

- Resultados de las evaluaciones

- Investigación en el aula para mejorar el aprendizaje por parte del estudiantados

- Participación en la comunidad científica local, nacional e internacional 
- Cumplimiento de la normativa universitaria

\section{¿Cómo debe ser?:}

- Respeto y consideración hacia de los y las alumnas

- Respeto de la Libertad de pensamiento de los demás (estudiantes, colegas)

- Capaz de escuchar y de comunicarse

- Con disposición para aprender del estudiantados

- Compromiso con su tarea docente

- Apertura al cambio e innovación

- Responsable en el cumplimiento de los tiempos y horarios

- Consciente de la relación entre la UCR y la sociedad

\section{La evaluación docente en los diferentes tipos de cursos:}

Las dimensiones y competencias anteriormente mencionadas constituyen un punto de partida para construir una evaluación docente. Sin embargo, es una caracterización muy general que requiere ser afinada y, con el fin de incorporar aspectos más específicos del desarrollo docente, se consideró pertinente identificar las estrategias de aprendizaje que más comúnmente se utilizan y aclarar tanto sus características como las especificidades docentes que se requieren en cada una de ellas.

En el contexto de la Universidad de Costa Rica, se revisaron las actividades de aprendizaje más comunes para consensuar una definición y ubicar en cada una de ellas las características particulares y las competencias específicas requeridas del personal docente, como aspectos a ser considerados en la evaluación docente.

Comúnmente se incluyen dos actividades de aprendizaje básicas, muy conocidas por la comunidad universitaria que son los cursos teóricos y los cursos prácticos. Se inicia por tanto a continuación la discusión de ambos, procediendo luego a aclarar una diversidad de formas de aprendizaje que toman esas actividades. Las definiciones que se presentan a continuación constituyen las aclaraciones brindadas por docentes que participaron en la investigación. En el caso de los cursos teóricos, éstos podrían reflejar un enfoque técnico academicista del docente, si bien se hace necesario obviar la dicotomía entre éstos y los prácticos para comprenderlos más bien en su complementariedad. 


\section{a. Los cursos teóricos y los cursos prácticos-}

Tradicionalmente, la docencia universitaria ha manejado el término de cursos teóricos como la actividad de aprendizaje en la cual los contenidos temáticos, las actividades del estudiantado y el papel del profesor o profesora se orientan a la comprensión de cuerpos estructurados de conocimientos, usualmente en forma de conceptos, teorías y principios científicos. Los cursos teóricos se centran fundamentalmente en la exposición, explicación, demostración y guía del docente para contribuir a que sus estudiantes comprendan los aspectos teóricos que se presentan y las actividades que se exigen al estudiantado privilegian las horas de aula dirigidas por un personal docente.

Por su parte, los cursos prácticos constituyen unidades de aprendizaje en las cuales los contenidos temáticos, las actividades del estudiantado y la función docente se orientan a la adquisición, por parte del estudiantado, de habilidades y competencias propias de la aplicación del conocimiento, así como a la solución de problemas, la creación de artefactos, físicos o lógicos, la interpretación de situaciones o la verificación de principios o leyes. Los cursos prácticos demandan del estudiantado participación activa, utilización de equipos o ubicación de situaciones de aplicación práctica de conocimientos y habilidades específicas.

Este segundo tipo de cursos demandan del profesorado la planificación de actividades, la demostración y sobre todo la observación, corrección y guía de la actividad práctica que realiza el estudiantado. Estos cursos demandan tiempo de ejercitación y aplicación de procedimientos, en situaciones cercanas a la realidad profesional.

Tanto los cursos teóricos como los prácticos no pueden considerarse como conceptos opuestos o dicotómicos pues el conocimiento teórico, para ser significativo, debe ser a la vez funcional y situado y el estudiantado ha de ser capaz de saber cómo funciona ese conocimiento en las diversas situaciones reales de su profesión en las que se puede aplicar y cobrar así sentido. Los cursos prácticos, a su vez, demandan, además de las competencias prácticas o de aplicación, claridad de conceptos teóricos básicos y criterios para discernir, en cada paso del proceso, qué principios o reglas se han de aplicar y por qué, para obtener determinados resultados.

Se puede caracterizar, en general, un grupo de cursos que exigen del estudiantado un proceso largo de creación, en el cual debe poner juntos aprendizajes y competencias 
diversas, encontrando soluciones a problemas complejos o diseñando creativamente producciones originales. En estos casos, el profesor o profesora actúa como persona que orientada, facilita y guía el trabajo estudiantil. Estos cursos favorecen la interacción entre estudiantes, la flexibilidad en las horas de contacto entre docente y el alumnado, según las necesidades durante el proceso. No tienen una denominación común sino que, a este grupo pertenecen los módulos de formación, los cursos por proyectos, los laboratorios, los denominados talleres en las áreas de artes o arquitectura, los proyectos colaborativos y tal vez las denominadas prácticas profesionales, cuando demandan una inserción del estudiantado en la comunidad de práctica real.

A continuación se explicitan algunos tipos de cursos, impartidos en la Universidad de Costa Rica:

\section{b. Cursos laboratorio:}

Son actividades de aprendizaje de carácter práctico. En ellos el estudiantado aplica conocimientos y practica habilidades y destrezas mediante la realización de experimentos, análisis de fenómenos, verificación de principios o modelos, cuya realización requiere la utilización de instalaciones, instrumentos y equipos especializados.

En cuanto al desempeño docente, el profesorado que participa en cursos laboratorio debe ser capaz de: planificar y disponer previamente lo necesario para las actividades, demostrar un uso adecuado de los equipos y materiales, contextualizar la actividad en el ámbito de la profesión, guiar y facilitar la ejecución de las actividades, analizar y evaluar el desempeño del estudiantado.

Para la Universidad de Costa Rica, existen a la vez una serie de especificidades que se deben considerar, entre las que se encuentra:

Para los laboratorios de las Ciencias Biomédicas: El profesorado utiliza como estrategias, formar en el estudiantado el cumplimiento estricto de las normas éticas, normas de bioseguridad, normas de manejo responsable de sustancias peligrosas, así como su propio comportamiento al respecto, y la capacidad de trabajar en equipo. 
Por su parte, para las carreras de Lenguas Modernas los laboratorios son cursos prácticos que utilizan intensivamente medios de audio o video interactivos, con la guía docente.

En las carreras de Computación y de Informática, el laboratorio no cumple estrictamente las características de la definición de laboratorio y es más bien un curso práctico.

\section{c. Curso Taller:}

Es una actividad de aprendizaje de carácter práctico, en la cual el estudiantado realiza trabajos sobre una realidad concreta y son sujetos u objetos propios de la acción profesional, siendo de particular importancia el proceso de solución o ejecución, en el cual el estudiantado debe aplicar en forma integrada conocimientos y destrezas, siguiendo el principio de aprender haciendo; se privilegia el trabajo grupal, con síntesis individuales.

En este tipo de curso se requiere que el personal docente sea capaz de: planificar e informar con claridad los objetivos de aprendizaje, los procedimientos y los criterios para juzgar la calidad del proceso y de los resultados; guiar el trabajo individual y coordinar el trabajo grupal; dominar técnicas de diálogo, preguntas y respuestas y de manejo de conflictos; mantener apertura y flexibilidad para aceptar respuestas divergentes y favorecer así la creatividad; ofrecer correcciones oportunas e información de retorno permanente ajustada a los requerimientos del estudiantado.

Los cursos de taller ofrecidos en la Universidad de Costa Rica tienen las siguientes especificidades:

Para la carrera de Arquitectura, el taller es el elemento central del currículo, transversal a lo largo de la carrera. Se debe evaluar el desempeño del profesor en el trabajo colegiado; su capacidad de interrogar críticamente y de orientar al estudiantado durante el proceso de análisis y diseño de soluciones; su capacidad para mantener un equilibrio entre una dirección y control más intenso en las etapas iniciales y una progresiva autonomía del estudiantado en etapas posteriores; su capacidad de transmitir al estudiantado un modelo integral de profesional de la arquitectura. 
Para la carrera de Trabajo Social, el taller se traduce en la aplicación de técnicas de investigación e intervención en realidades sociales grupales, comunales, organizativas o de proyectos sociales. El docente debe ser evaluado como planificador de las actividades, incluyendo los aspectos logísticos que garanticen una ejecución eficaz; su capacidad de interactuar con grupos externos; su capacidad de solución de conflictos. Debe tener habilidades para relacionarse con los centros de práctica

\section{d. Práctica profesional}

Como su nombre lo indica, este es un curso de carácter práctico, de larga duración. Consiste en la participación del estudiantado en una experiencia de aplicación de su especialidad disciplinar. El estudiantado se inserta en el ámbito profesional e interactúa con la realidad social. Esta actividad le ofrece la oportunidad de investigar el contexto y desarrollar competencias multidisciplinarias al integrar la teoría y la práctica. Se espera que demuestre un alto nivel de compromiso con el proceso, capacidad de priorizar y jerarquizar para la toma de decisiones acertadas, y habilidad comunicativa para vincularse con las instancias externas públicas y privadas

Para este tipo de curso se requiere que el docente y la docente a cargo muestre estas competencias: ser mediador con el actor social, establecer los vínculos y facilitar las condiciones para que se den las prácticas; tener habilidades para comunicarse eficazmente, respetuoso con las instancias e instituciones que prestan sus instalaciones; tener experiencia en el campo profesional y disposición para realizar cambios e innovaciones; asesorar y evaluar el desempeño del estudiantado; definir con claridad cuales son las intenciones de la experiencia, en qué condiciones se realiza la práctica y que responsabilidad tiene cada uno. En la evaluación se hace necesario considerar también aspectos relacionados con la cooperación entre el actor social y la UCR.

\section{d. Seminario}

Es una actividad de aprendizaje de carácter teórico. En ella el estudiantado está orientado a fomentar el trabajo en equipo y el aprendizaje autodirigido. Los participantes toman parte activa en el desarrollo del curso junto con el profesor que dirige y coordina sobre la base de lecturas críticas, investigaciones, bibliografía consultada y el conocimiento previo de cursos anteriores. Estos pueden ser temas específicos de la disciplina o intereses particulares. Se espera desarrollar en el estudiantado el hábito de la lectura con actitud 
crítica, fortalecer las habilidades de la escritura y de la expresión oral, la discusión y el respeto en la opinión fundamentada no sobre la base de cualquier ocurrencia, la búsqueda de la honestidad intelectual y la creatividad

En este tipo de curso, se espera que cada docente sea capaz de: coordinar, orientar y evaluar la discusión y la exposición de los alumnos; manejar técnicas de trabajo grupal para generar la participación de los estudiantes; ser respetuoso con las diferencias y la libertad ideológica; tener habilidades para investigar; sintetizar los argumentos tratados en el aula, demostrando ser un conocedor de la temática. Cuando el seminario es colegiado, se recomienda realizar una evaluación entre colegas del desempeño conjunto de los docentes

\section{e. Módulo:}

Es un curso de tipo teórico-práctico. El curso tipo módulo enfrenta al estudiantado a un conjunto de actividades de aprendizaje alrededor de un eje temático, problema u objetivo, que privilegia la práctica y la integración de conocimientos, fortaleciendo el carácter interdisciplinario y multidisciplinario de la organización curricular. Permite al estudiantado fomentar el trabajo en equipo, el espíritu de investigar e interpretar la realidad social, mejorar la capacidad reflexiva, crítica y analítica. Desarrolla la capacidad de liderazgo, de autonomía y creatividad bajo un comportamiento ético y responsable de sus actos

Para este tipo de curso se espera que el profesorado sea capaz de: comprometerse ética y socialmente con la tarea; manejar metodologías participativas que orienten a los estudiantes al abordaje temático; saber intervenir en un campo específico ante problemas definidos; planificar e informar los objetivos de aprendizaje, los procedimientos y los criterios para juzgar la calidad del proceso y de los resultados; dar apoyo y acompañamiento al trabajo individual y coordinar el manejo de trabajo en grupo.

\section{Conclusión:}

Esta investigación se une al cúmulo de conocimientos que tiene la Universidad de Costa Rica en relación con su larga trayectoria de evaluaciones de su personal docente, en cuanto a su labor en el aula.

La evolución conceptual de la evaluación, desde un enfoque técnico e informativo hacia procesos evaluativos de carácter formativo, que generen la autorreflexión, el Volumen 5, Número Especial, Año 2005, ISSN 1409-4703 
autoaprendizaje y la autogestión en el personal docente y en las unidades académicas, provoca la necesidad de establecer un sistema de evaluación docente en la Universidad de Costa Rica con diferentes fines, en donde se integren enfoques evaluativos diversos, de acuerdo con los diferentes usos asignados a la evaluación.

En este artículo se han presentado los logros obtenidos de una investigación que pretendió, en una primera etapa, la aclaración de lineamientos generales que permiten aclarar las competencias docentes, con respecto a las funciones esperadas del y la docente en la Universidad de Costa Rica según la diversidad de cursos que se ofrecen en la institución. Esta información servirá para nutrir el diseño de un sistema de evaluación docente capaz de brindar información para diferentes finalidades tales como contribuir al conocimiento de la capacidad instalada de la institución, realimentar la toma de decisiones de la administración central y de las diversas unidades académicas, fortalecer procesos de autoevaluación-autorregulación, detectar aspectos para la capacitación docente y brindar información para el ascenso del personal docente que se encuentra incorporado al Régimen Académico.

En aras de construir una cultura evaluativa en la institución, se evidencia también la necesidad de establecer espacios y actividades formativas que permitan pasar de un concepto tradicional y controlador de la evaluación hacia el uso de procesos evaluativos capaces de ser utilizados para el autoconocimiento y el mejoramiento continuo del personal docente. De esta forma, se podría ejecutar un sistema de evaluación, caracterizado por ser sistemático, específico y equitativo para el personal académico, que pondere y valore lo académico, según las funciones de cada docente, reconociendo la especificidad de su trabajo y que permita planificar acciones, con miras al mejoramiento del proceso académico en el ámbito institucional. La aclaración de la labor idónea que debe realizar el personal docente en términos generales y específicos y de identificar competencias y desempeños que se consideran como excelentes en el quehacer docente es un paso necesario pero no suficiente para la .puesta en marcha de procesos evaluativos realizados por diferentes actores según sus diversos fines.

El principal aporte de este estudio ha sido la aclaración del conjunto de atributos que la comunidad universitaria señaló como propios de una buena docencia, tanto en términos generales como de acuerdo con los tipos de curso que se imparten en la institución. Esto 
permitió aclarar la caracterización de las competencias docentes e identificar las condiciones específicas de ese desempeño, propias de cursos de tipo particular, lo cual es imprescindible de considerar al evaluar su calidad. Se pretende que la lectura de este artículo brinde elementos para dimensionar una buena docencia y construir, a partir de lo aquí expuesto, nuevas formas de evaluar al personal docente de manera sistemática, específica y con miras al mejoramiento del proceso académico en el ámbito de la educación superior 


\section{Referencias}

Apple, M. W. (1996). Política cultural y educación. Madrid, España: Ediciones Morata, S.L.

Brunner, J. J. (1993). Educación superior en América Latina durante la década de los ochenta: La economía política de los sistemas. Buenos Aires, Argentina: Centro de Estudios de Estado y Sociedad (CEDES), Serie Educación Superior 2.

De Alba, A. (1991). Evaluación curricular. Conformación conceptual del campo. México: Universidad Nacional Autónoma de México, CESU.

De Alba, A. (1993). Currículum: crisis, mito y perspectivas. México: Universidad Nacional Autónoma de México, CESU.

Díaz-Barriga, A. (1995). Empleadores y universitarios: un estudio de sus opiniones. México: Universidad Autónoma Nacional de México. CESU.

Díaz-Barriga, A. y Pacheco, T (Coordinadores) (1997). Universitarios: Institucionalización académica y evaluación. México: Universidad Nacional Autónoma de México, CESU.

Giroux, H. A. (1997). Cruzando límites. Trabajadores culturales y políticas educativas. Buenos Aires, Argentina: Ediciones Paidós Ibérica, S. A.

Jiménez, B. (1999). "Evaluación de la docencia". En: Jiménez, B. et al: Evaluación de programas, centros y profesores. Madrid: Síntesis Educación

Llarena de Thierry, R. (1991). Evaluación del personal académico. En: Perfiles Educativos, (53-54), 18-28.

Scriven, M. (1997). Selección de profesorado. En: Manual para la evaluación del profesorado. Millman, J. y Darling-Hammond, L. Madrid: Ed. La Muralla.

Universidad de Costa Rica. (2002). Reglamento de Régimen Académico y Servicio Docente. Recuperado el 3 de marzo 2005, de http://cu.ucr.ac.cr/regñlamen/r1451$\underline{\text { 06.html }}$

Yarzabal, L. (1999). Globalización neoliberal y educación superior. En: Universidad iberoamericana. Globalización e identidad. Colección ExtremAmérica (pp. 25-34). Madrid, España: Julio Le Parc. VEGAP. 